\title{
Mobility assistance for older people
}

\author{
Daniel Eck ${ }^{\mathrm{a}, *}$, Klaus Schilling ${ }^{\mathrm{a}}$, Ahmad Abdul-Majeed ${ }^{\mathrm{b}}$, Jörn Thielecke $^{\mathrm{b}}$, Phillipp Richter ${ }^{\mathrm{c}}$, \\ Javier Gutiérrez Boronat ${ }^{\mathrm{c}}$, Irina Schens ${ }^{\mathrm{d}}$, Bernd Thomas ${ }^{\mathrm{d}}$, Bettina Williger ${ }^{\mathrm{e}}$ and Frieder R. Lang ${ }^{\mathrm{e}}$ \\ ${ }^{a}$ Computer Science VII, University of Würzburg, Würzburg, Germany \\ ${ }^{\mathrm{b}}$ Department of Electrical, Electronic and Communication Engineering, LIKE, University of Erlangen-Nuremberg, \\ Erlangen, Germany \\ ${ }^{\mathrm{c}}$ Power Efficient Systems Department, Fraunhofer Institute IIS, Nuremberg, Germany \\ ${ }^{\mathrm{d}}$ R\&D Pre-Development, NAVIGON AG, Würzburg, Germany \\ ${ }^{\mathrm{e}}$ Institute of Psychogerontology, University of Erlangen-Nuremberg, Erlangen, Germany
}

\begin{abstract}
This paper describes the development of a scooter supporting the mobility of older people. The scooter is equipped with a drive assistance system and a special scooter navigation system. The drive assistance system consists of a velocity controller, a steering controller, and a collision avoidance system. In this paper it is demonstrated how the challenging control and steering tasks are modified to increase safety for older people. A special scooter navigation system is presented, to support elderly people in navigating on a safe route through the city using sidewalks, pedestrian lights and crosswalks. For extended positioning requirements a hybrid positioning system was developed combining GPS, WLAN, and inertial sensor data. By combination of these technical improvements it is demonstrated how older people are able to preserve their self-determined and independent life. Usability research was done with focus groups in order to become familiar with global user demands and expectations towards a mobility assistance system. Results show that the system components are expected to assist the user in navigation, steering and speed control rather than to take complete control on the driving situation.
\end{abstract}

Keywords: Navigation, mobile robot, scooter, positioning, WLAN

\section{Introduction}

Mobility is one of the biggest issues for aging generations. For most older people mobility is a critical prerequisite of social participation and instrumental activities of daily living (IADL) such as shopping, errands or doctor visits [33]. Consequently, mobility is critical to maintaining autonomy and selfdetermination in old age. In Germany, the proportion of people aged over 65 years in the whole population will increase from 16 percent in 2000 to more than 25 percent in 2030 [9]. Considering the cognitive and physical losses that are often associated with normal

*Corresponding author: Daniel Eck, Computer Science VII, University of Würzburg, Am Hubland, 97074 Würzburg, Germany. E-mail: eck@informatik.uni-wuerzburg.de. aging processes, there are a number of challenges and potentials regarding the maintenance of independence and high levels of life quality in old age. We define geographic and physical mobility as moving independently and safely from one place to another [32]. Any loss or decline of such mobility posits threats to the individual's everyday competence and maintenance of autonomy in later life. Innovative, resource-protecting and easy-to-use technical assistance in physical mobility provides much potential for vitality and autonomy of older populations in an aging society.

The present study aims at developing a tool that can easily be adapted to changing needs of older people, who find themselves in a critical transition from normal to pathological aging with more accelerated declines in IADL and other capacities related to mobility. The vehicle should support older users in their daily life 
(e.g. shopping, doctor visits). Because of the challenging control of such a vehicle, we will integrate drive assistance functions (like a velocity controller and a collision avoidance system) and a navigation system. The later shall be adapted to pedestrian issues, to be able to navigate the older persons on a safe route to their target, using sidewalks, pedestrian lights and crosswalks.

As for navigation a very accurate positioning of the vehicle is necessary a fusion of GPS and a WLAN positioning system is presented to get an exact position. All these systems were integrated on the scooter. The positioning system is connected directly to the navigation device and will provide the current position continuously. The navigation device is mounted directly in front of the user, above the steering wheel. The fixture can be seen in Fig. 1.

While pure mechanical supports, like wheeled walkers, also called rollators, are becoming popular due to their small costs, more "intelligent" mobility assistance systems are still in an early stage of development. Electrical wheelchairs impose control challenges for young as well as older users [34]. In a driver competency test with elderly people more than $65 \%$ of the participants failed at least at one test item [25]. Therefore, assistance systems are necessary to support the operator and ensure a safe control. However, more elaborate systems, providing assistance functions are typically targeting at more severely handicapped people [29, 11]. Research activities focusing on electrical wheelchairs address sensor based support to warn about invisible obstacles [20,23] or to inform service centres in case of emergency about the position of the vehicle and crucial health parameter of the user [31]. Already existing mobility assistance systems are mainly for indoor usage [30, 28, 3, 17]. Additionally none of these systems are equipped with a global navigation system. In general, commercially available outdoor wheelchairs are not equipped with assistance systems [6]. Nevertheless, there exists small rovers with drive assistance systems [7], but they are primarily used for tele-operation. The focus of the presented work is to transfer these drive assistance systems to a commercially available mobility scooter.

Parts and demonstrators of a scooter device with integrated navigation and orientation systems were developed and also evaluated within a group of healthy older adults. Such user studies allowed for a more in-depth understanding of evaluations and attitudes among a potential target user group.

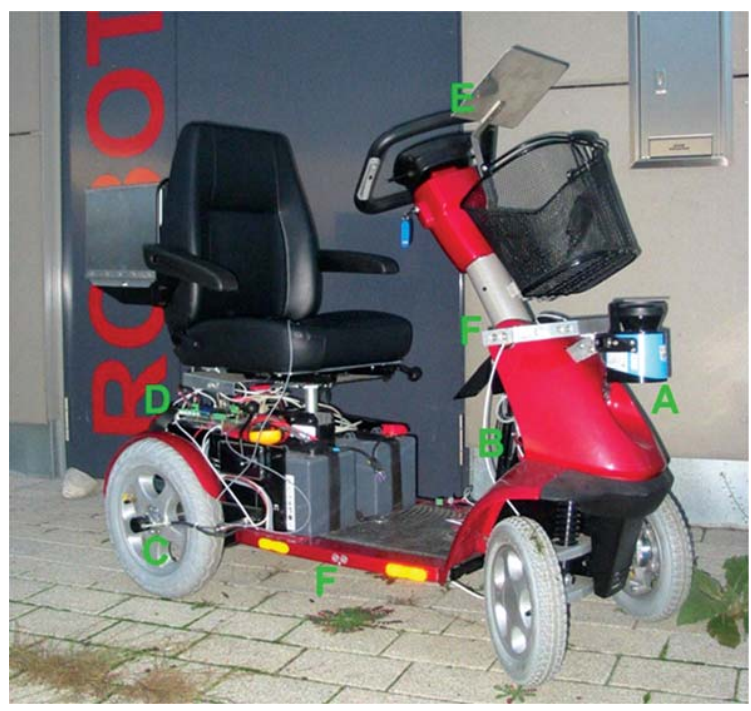

Fig. 1. The mobility scooter equipped with all electronics and sensors A) Laser Range Finder, B) steering motor, C) incremental sensor, D) electronics, E) mounting device of the navigation system, F) ultrasonic sensors.

The paper is organized as follows. The next section introduces the vehicle, the modification and the drive assistance functions on the vehicle. The positioning system will be explained in section III. Afterwards, the navigation system will be presented. Tests of the system in focus groups will follow. Finally, the last section will summarize this paper and point out the future work.

\section{The mobility scooter}

This section describes the robotic scooter starting with an introduction of the basic scooter vehicle. The integration of the microcontrollers, the sensors and the drive assistance systems will follow.

\subsection{The basic mobility scooter}

The car-like scooter Trophy 4W $\left({ }^{\circledR}\right.$ Handicare $\mathrm{GmbH}$ ) is used as basis (see Fig. 2). The scooter meets the standards of the German MOT approval, hence it is possible to drive on public streets. It can drive up to $15 \mathrm{~km} / \mathrm{h}$ covering a distance of $60 \mathrm{~km}$. Only the rear axis is actuated by the electric motor. The steering must be done by the operator. 


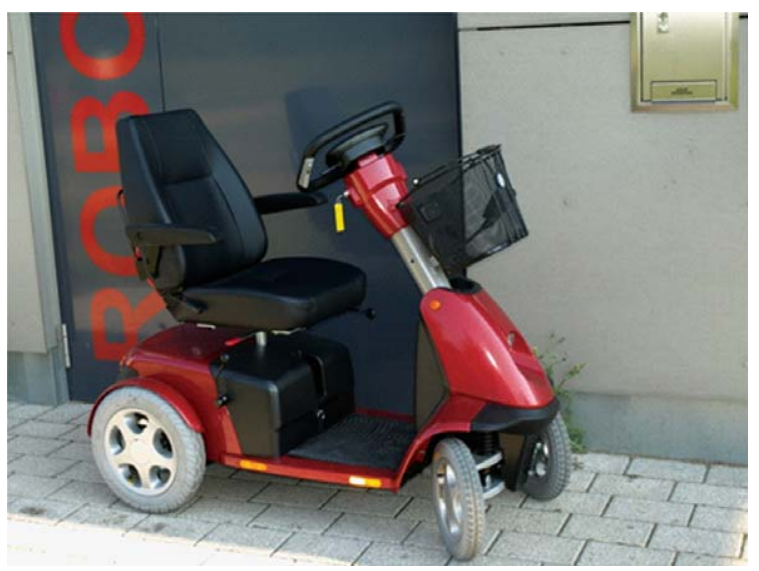

Fig. 2. The basic scooter vehicle.

Figure 3 displays an overview of the onboard electronics. The original electronics on the mobility scooter consist of three main parts: the tiller printed circuit board (PCB), the scooter control box (SCB) and the motor controller (blue boxes on Fig. 3). The tiller PCB is mounted in the steering wheel and offers all interfaces to the operator. The lights and the horn can be activated or deactivated via this interface. Battery status, the current speed and the covered distance are indicated on a display. Additionally a hand throttle is mounted at the steering wheel. All devices on the mobility scooter (like lights, horn, tiller PCB, motor controller, and batteries) are connected to the SCB. The SCB switches these devices on and off, when they are needed, when they are needed. If there is no error state, the SCB will forward the driving commands of the operator to the motor controller.

\subsection{Hardware and software extensions}

The scooter Trophy $4 \mathrm{~W}$ offered no interface to control the scooter via a PC or microcontroller. Hence, it was necessary to find some interface in the electronics to be able to control the motor and to use all available functionalities on the scooter. Therefore, a small microcontroller AT90CAN128 from ATMEL was integrated in the scooter to wiretap the connection between the tiller-PCB and the SCB. Thus all commands of the operator can be received by the microcontroller. These commands can be forwarded to the SCB or can be processed at first and forwarded afterwards. The SCB was not able to distinguish between the original commands generated by the tiller PCB and the commands processed or generated by the microcontroller. By the means of this new interface it was possible to activate and deactivate all devices and to control the

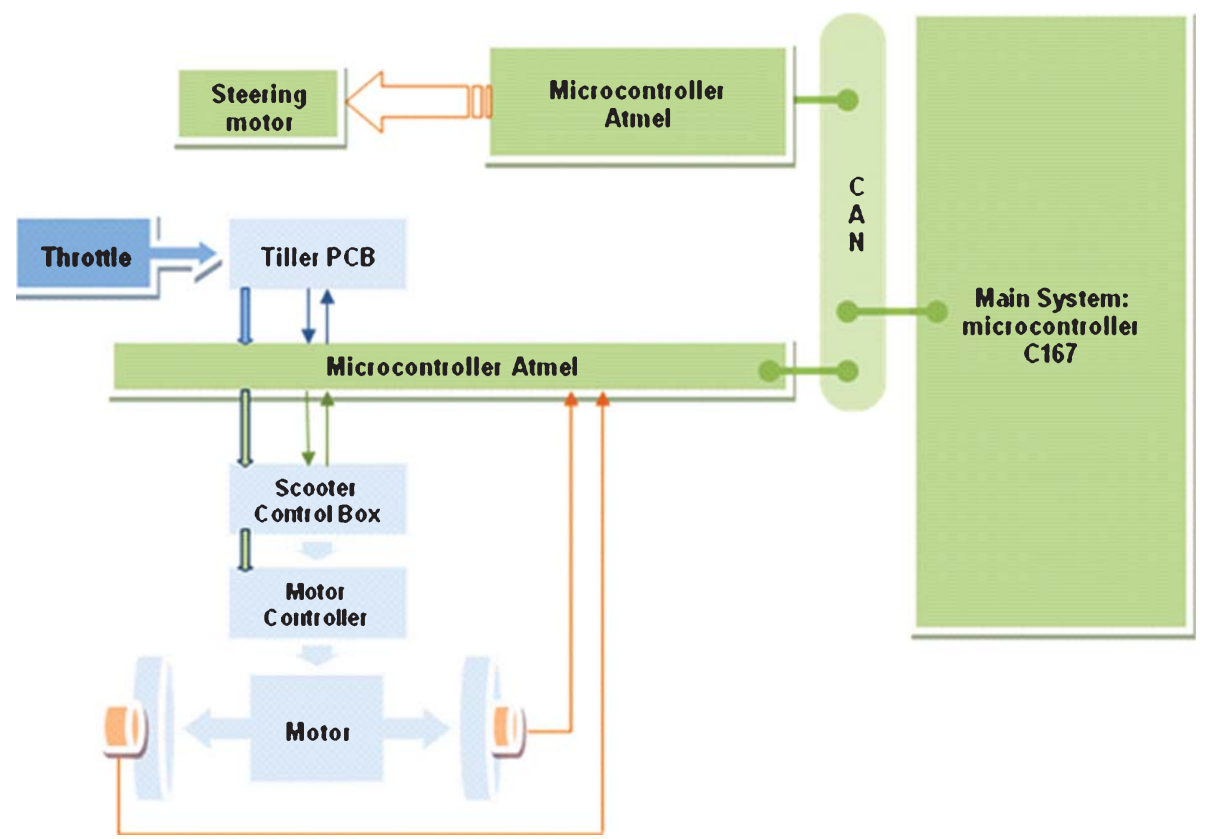

Fig. 3. The scooter electronics and the extensions (blue: the origin electronics, green: additional mounted electronics). 
motor as well. Furthermore, two incremental sensors were connected to this microcontroller. The incremental sensors measured the round per minute of both rear wheels. Thus, the velocity of the vehicle can be determined. By the use of the measured velocity a closed loop controller for the driving motor was enabled. The characteristics of the motor and the mobility scooter were determined in driving tests. Therefore, the vehicle was accelerated from 0 on a flat basement and at a slope. During these test runs, the current velocity, the driving commands and the time stamp were recorded. According to the results of the test runs, a simulation of the mobility scooter was implemented with Matlab. Using this simulation various closed-loop controllers were tested to find an appropriate one. The best simulation results were achieved with a PI controller. However, in the simulation the model of the mobility scooter was approximated. For this reason, a PI controller was implemented on the vehicle to evaluate the results of the simulation on the vehicle. The same test runs as before were accomplished and the according measurements were recorded in order to validate the parameters with Matlab again. Thus, the parameters had to be modified a bit to get an adequate velocity controller for the mobility scooter Trophy $4 \mathrm{~W}$. The PI controller was used for autonomous functions and for the manual mode as well. Due to the velocity controller it was easier to control the mobility scooter especially at acclivities and the operator is partially relieved of the challenging control.

To enable autonomous functions, a steering motor had to be mounted on the mobility scooter, because only a driving motor was integrated in the original scooter. The steering motor was chosen according to the maximum measured steering force. Two different control-loops (force and position control) were offered by the steering motor controller to operate the motor. The current steering angle of the motor was measured with a hall sensor. Using this hall sensor it was possible to detect changes of the steering angle even if the scooter was deactivated. A second AT90CAN128 microcontroller was integrated to control the steering motor and to evaluate the hall sensor data. The position control loop of the motor controller can be applied using the hall sensor measurement. Thus the motor can be run into every desired steering angle.

The high level functions and algorithms are implemented on a third more powerful microcontroller C167. This microcontroller offered a lot of additional interfaces like RS232 or I2C for further sensors. The
C167 is connected to both ATMEL-microcontroller via a CAN bus. All driving and steering commands of the high level functions are generated on the C167.

A collision avoidance system was integrated as a drive assistance system to facilitate of the challenging operating of the scooter for the older persons (see section User Studies) [8]. The collision avoidance system is based on ultrasonic sensors and a laser range finder (LRF) (see Fig. 1). A total of twelve ultrasonic sensors were mounted on the scooter: four at the front, three on each side and two on the rear. The LRF was mounted in front of the vehicle. Obstacle zones around the vehicle were defined according to the measurement areas of the ultrasonic sensors. Detected obstacles are matched to these zones. A finite state automation controls the velocity of the scooter according to detected obstacles. This automation monitors only the zones in the driving direction. If there is one obstacle in driving direction of the scooter, the finite state automation reduces the velocity linearly and stops the scooter 20 $\mathrm{cm}$ in front of the obstacle. For more information about the automation refer to [8].

From a technical point of view it was possible to integrate the steering motor for a collision avoidance system and to drive around obstacles autonomously. However, the response results of the evaluation study indicated that users may generally prefer that the vehicle decreases the velocity instead of bypassing the obstacles autonomously. Thus, the driver may decide on his own when and in what direction they want to steer the mobility scooter around the obstacle. More details about the evaluation of the scooter in the focus groups are given in "User Studies".

All these integrated driving assistance function were activated all the time. The user cannot disable one of these. For this reason, no special human-machineinterface was integrated on the mobility scooter. The autonomous functions are closely linked to the navigation system and can be controlled via the navigation device (see section "Navigation").

\section{Global positioning}

Over the past years, new applications based on location information, the so-called Location Based Services (LBS) have rapidly penetrated markets worldwide. Most of such products rely on the well-extended navigation systems based on the Global Position- 
ing System, GPS, or on the localisation in mobile communication networks. By means of LBS it is thus possible to check up anywhere and anytime for a pharmacy, toilets, shops, a restaurant, available real states [18] or anything else. But all this is possible if the user's current position is accurately known. Generally GPS receivers do not work indoors and are not optimally suited for pedestrian navigation in urban environments. Often they lack an appropriate motion model and they have not the ability to suppress errors due to multipath propagation, which are common in urban scenarios. Another disadvantage is that the heading information from GPS receivers cannot be trusted at the low pedestrian speed. To solve these problems, a GPS receiver is enhanced with supporting positioning systems, namely a Wi-Fi based positioning system and an inertial navigation system.

\subsection{Localisation system classification}

A very important aspect when handling with location information is to guarantee the privacy of the user for the acceptance of these services. So it is important to point out that while a GPS navigation system performs the localisation on-board, the mobile network based locating systems often perform the localisation off-board, it means inside the network. Afterwards the position has to be transmitted to the handheld or mobile unit. This means that third parties can collect information about user's habits. Furthermore GPS is only available outdoors, which makes it hardly applicable for a number of scenarios like indoor or indoor-similar rough environments like urban canyons.

A matured GPS-like alternative technology to determine the own position on-board, i.e. without getting the own position information through third parties, is the WLAN-Positioning-System, shortly WPS, as the awiloc ${ }^{\circledR}$ system developed at Fraunhofer Institute IIS in Nuremberg [1, 21, 22, 24]. WLAN stands for Wireless Local Area Networks and it is also frequently known as Wi-Fi. The WPS achieves a precision similar to GPS. Since WPS uses the existing WLAN infrastructure it has no additional costs for the endusers, who need a device, which is only equipped with WLAN. However, rather than GPS it is possible to use WPS within buildings. Applications using WPS were becoming more important within the last years as shown by products like MobileWalk [13], which is successfully tested in real environments.

\subsection{Approach: Seamless positioning by means of GPS, WPS and INS}

Since GPS, WPS, and the inertial sensors all have complementary characteristics and are present in plenty of commercial PDAs, smartphones and some PNAs, a combination of these sensors allows a continuous navigation with low costs for the users. This work presents an approach to combine three complementary positioning systems. As mentioned before GPS shows its strength in outdoor environments. Moreover, the GPS-positions are quite noisy, presenting high standard deviation, but during the long-time they remain constant. The WPS-positions behave similar to GPSpositions from the previous point of view but WPS offers positioning in the areas where GPS begins to have difficulties, i.e. as indoor-like environments (e.g. in urban canyons) or even indoors. The inertial sensor data exhibits accurate measures in the short-time but the calculated position error rises to the cubic power of time. Thus it can be seen that the three systems offer complementary characteristics. By adding information from inertial and magnetic sensors it is possible to enhance the calculated position and what is more to determine the own heading without the constraint of having to be in motion. Using stand-alone GPS or WPS requires users to be moving to get meaningful heading information. An additional problem, when using modern automotive GPS-Chipsets for pedestrians, is that they often freeze the position when motion is slowed down under about $5 \mathrm{~km} / \mathrm{h}$. Moreover, similar to an assisted-GPS, with the WLAN-based localisation, it is also possible to reduce the initialising time before getting the first valid position as with a stand-alone GPS.

By means of an interacting multiple model (IMM) algorithm [2, 4, 5, 19, 27] running several Kalman filters [14] representing the different systems, it is possible to achieve a seamless localisation system. Figure 4 shows the implemented architecture in this work for the IMM algorithm.

The IMM-algorithm implementation in this work processes the position and quality of the GPS and WPS sensors and decides autonomously, after an evaluation based on the likelihood and the prior model probability, which sensor provides reliable data. According to the model probability the position data of GPS and WPS are mixed and a new prediction is performed to provide an improved position estimate. For an appropriate performance of the sensor weighting process in the IMM-algorithm, proper 


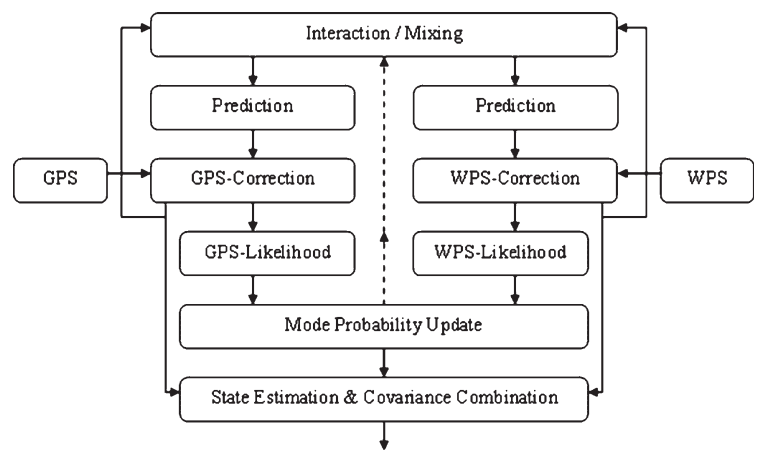

Fig. 4. Block diagram of the IMM algorithm.

quality parameter to describe the accuracy of the GPS and the WPS measures were to be defined. For the awiloc $^{\circledR}$ (WPS) system it is quite easy to monitor the operation of the system and thus to define a measure of the quality, since the system has been completely developed at Fraunhofer IIS. Contrariwise, for the GPS, as a low cost standard commercial receiver was used for this work, only parameter relying on the information contained in the NMEA strings could be used. This means firstly information related to the estimated position and parameter like the Dilution of Position, DOP. This information made it difficult to properly characterise the GPS-receiver performance. Consequently some research was initiated thus with an alternative GPS-receiver hardware, offering access to the pseudorange measures, the so called raw data before the PVT-software, in order to define alternative quality parameter, to take into account early effects, due to multipath for example. More specifically, in the test areas described below was not always possible to monitor accurately the performance of the GPS, and thus to weight both positioning systems in an optimal manner. The IMM algorithm estimates which model fits better to the received data, based on the assumption of a linear and Gaussian process and system.

The objective is thus to allow the user (a pedestrian or a scooter-user) to navigate in a city between the different environments, i.e. between indoor and outdoor without changing between navigation systems. Finally, it is possible to determine its own heading angle during both motion and rest time by using inertial sensors.

\subsection{First results}

Some tests have been performed in the Nuremberg city under real conditions to show firstly the online

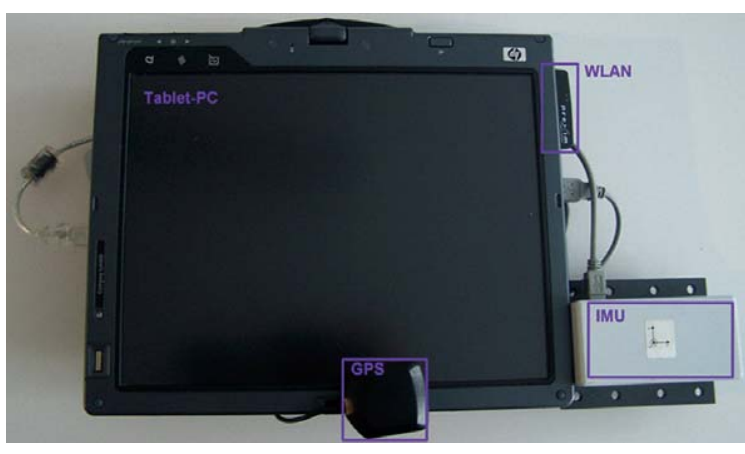

Fig. 5. Tablet-PC with the u-blox-GPS receiver, the WLAN-Card, and the IMU iSens-3.

feasibility of the implementation and secondly the indoor positioning advantages of the system. As development platform was chosen a standard tablet-PC, for the ease of the use. A new version for commercial standard handhelds is planned in the near future. The current system acquires the GPS-data with a commercial USB-GPS-receiver from u-blox [36]. The WLAN-Navigation was performed using the data for the Nuremberg city from the Fraunhofer IIS and an external PCMCIA-WLAN-Card to acquire additional data. Finally the iSens-3, an inertial measurement unit (IMU) developed at Fraunhofer Institute, was attached at one side of the tablet-PC. Figure 5 shows the development platforms as used in the tests which were conducted on the north side in the historic city centre of Nuremberg.

The true path of this test can be seen in Fig. 6 . Figure 7 shows the performance of the algorithm in an urban canyon scenario in a typical situation where multipath propagation is deteriorating GPS (blue) performance. It can be seen how the IMM-algorithm (green) is observing both solutions and weighting it. In this case weighting the WLAN-positions (cyan) more favourably and penalising the GPS ones it is possible to enhance whole performance.

\section{Navigation}

The availability and the use of mobile navigation systems has become familiar in everyday life for most people in modern western societies. Most navigation devices typically not only offer car navigation functionalities but also entail modes for user position tracking and pedestrian navigation. The market of 


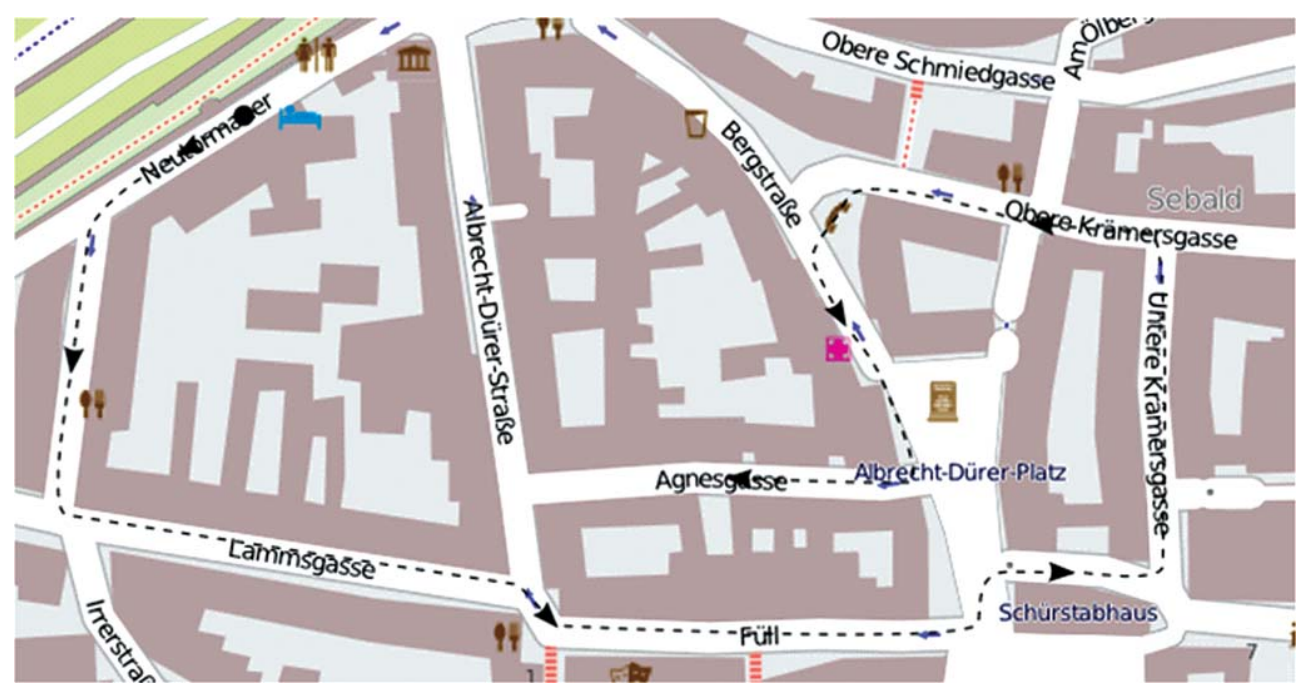

Fig. 6. True path (dashed line) of the localization system test in the north of city center in Nuremberg. Start point is in the upper left corner marked with a dot, end point in the center in "Agnesgasse" (OpenStreetMap).

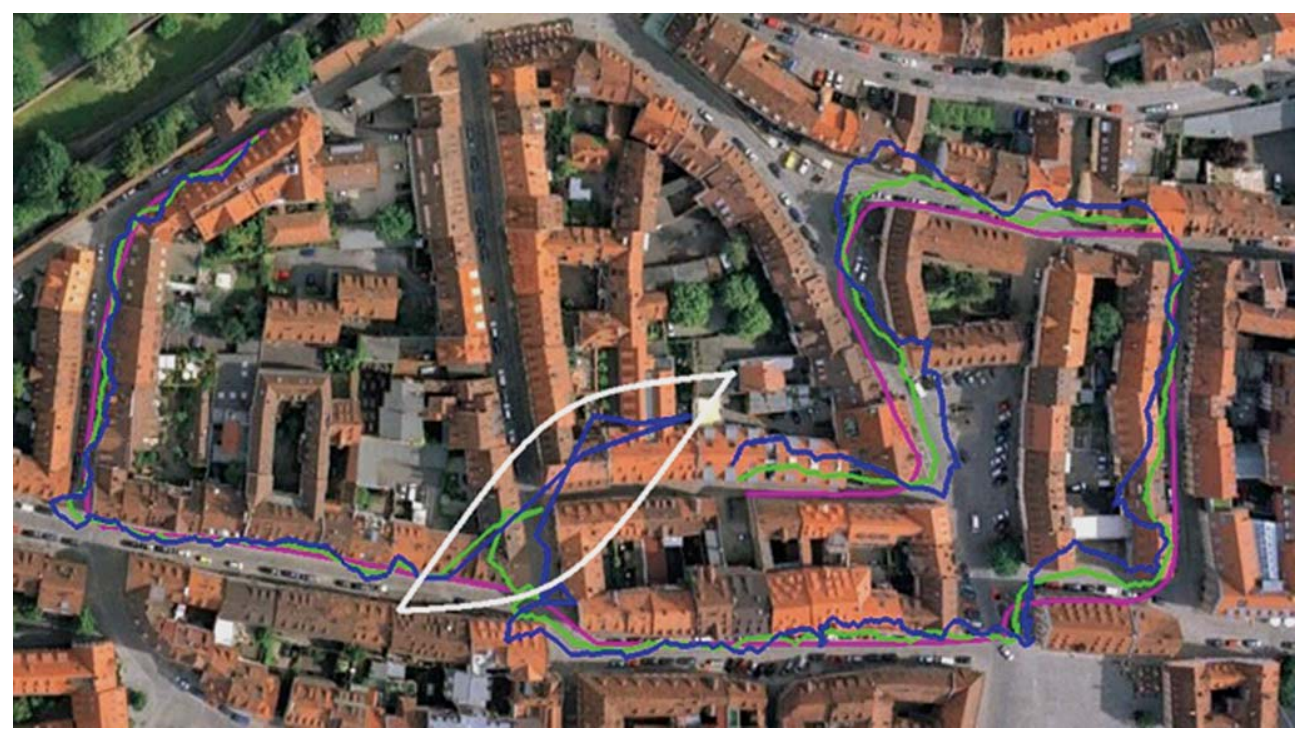

Fig. 7. GPS-Multipath in the city centre of Nuremberg and the better performance by means of IMM [GPS (blue); WPS (cyan); IMM (green)] (Google).

pedestrian navigation solutions is constantly growing, due to the ongoing development of GPS integration into mobile devices like smartphones and the increasing availability of digital map data in the context of pedestrian guidance.

However, most of these technical solutions are still based on the same map data model concepts as used in car-navigation systems. Extended and different data model concepts are required for pedestrian navigation tasks.
To improve the quality of these systems and to provide a pedestrian - friendly navigation, the NAVIGON AG examines different approaches. One of them focuses on extending digital maps with pedestrian specific attributes and the adaption and integration with existing navigation systems. Depending on additional digital map attributes and the different pedestrian navigation context in contrast to a vehicle centric navigation system, there are also modifications necessary regarding the route calculation components. 
Until now, most of the map data collection routines were designed for car-navigation systems. Such digital map models contain geometry information measured from the streets' central lines which is used for localization of the vehicle position. Geometry information and the current car position given by GPS are used to determine the vehicle position on the map. In addition to the mere geometry information many more attributes like street type classification, street names, lane info are usually contained in the digital map.

However, for the pedestrian navigation systems, different requirements for the geo-data collection should be applied. According to this, the currently available geo data sets were analyzed for the pedestrian navigation usability. The digital map data was checked for the "walk-ability" for the pedestrians. For example, it is not allowed for pedestrians to go on motorways or on highways. Hence reasonable constraints for the route calculation have to be defined. Furthermore, in contrast to car navigation systems special map attributes (like pedestrian zone, stairs, crosswalks, sidewalk etc.) should be included in the map for pedestrian navigation purposes. Extending street maps with pedestrian relevant attributes is not the only adaption to existing digital maps. Specific restrictions used for car navigation route calculation, for instance driving directions of streets, can usually be dropped for pedestrian route calculation as long as pedestrian sidewalks exist. On the other hand, the router must have an information on the existence of sidewalks (which side of the street it is located) and thus has to include the information in its route calculation to find save street crossings and traversal along dangerous roads.

Findings gained from the investigations and requirements analysis were used for the new map attributes definitions and corresponding concept design for the pedestrian suitable route planner. Supported by a commercial digital map supplier relevant pedestrian map attributes were collected and digitized for a test area in the city center of Würzburg. Figures 8 and 9 show the test area with and without additional pedestrian ways. Additional pedestrian attributes like

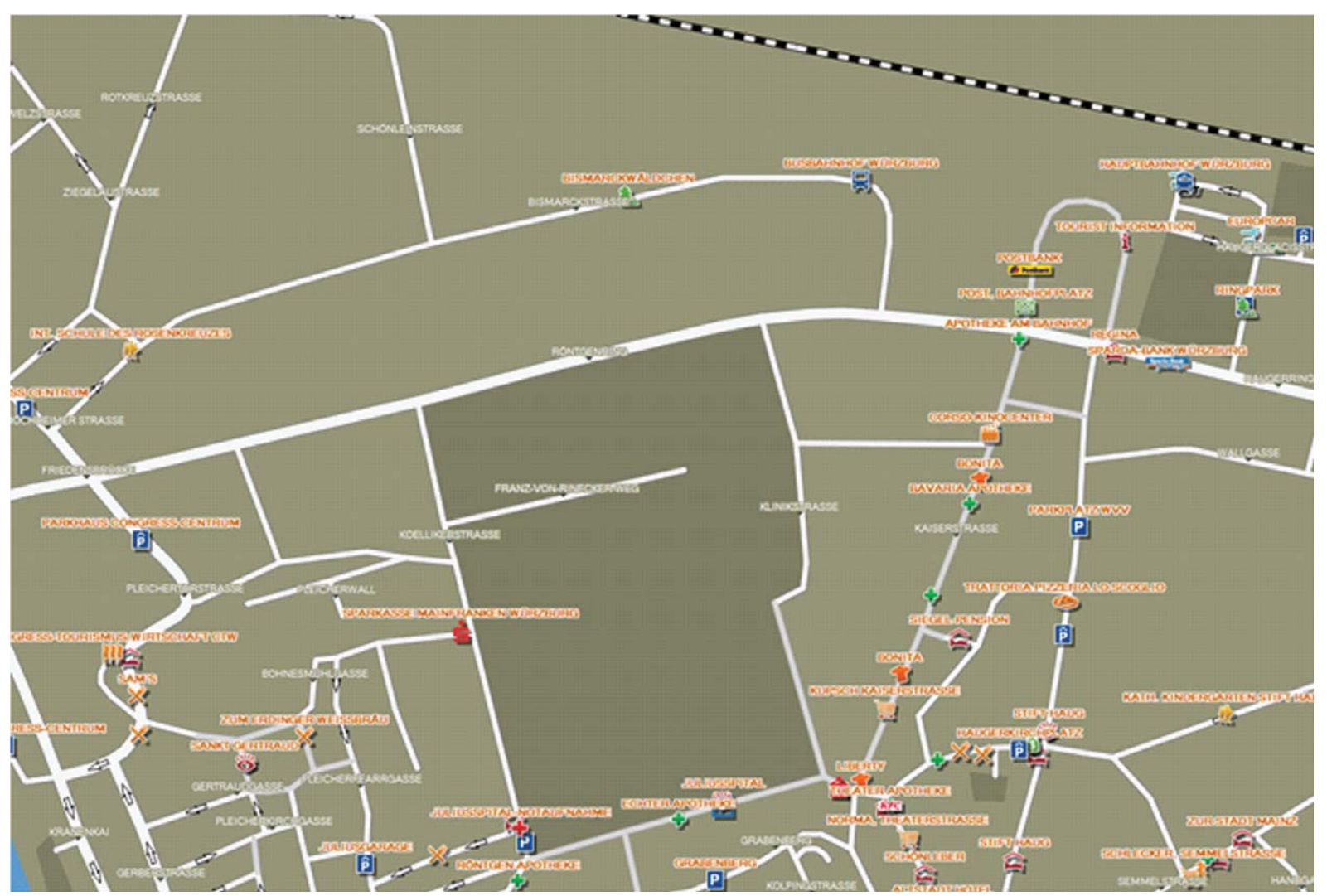

Fig. 8. Part of the test area Würzburg: map without pedestrian ways. 


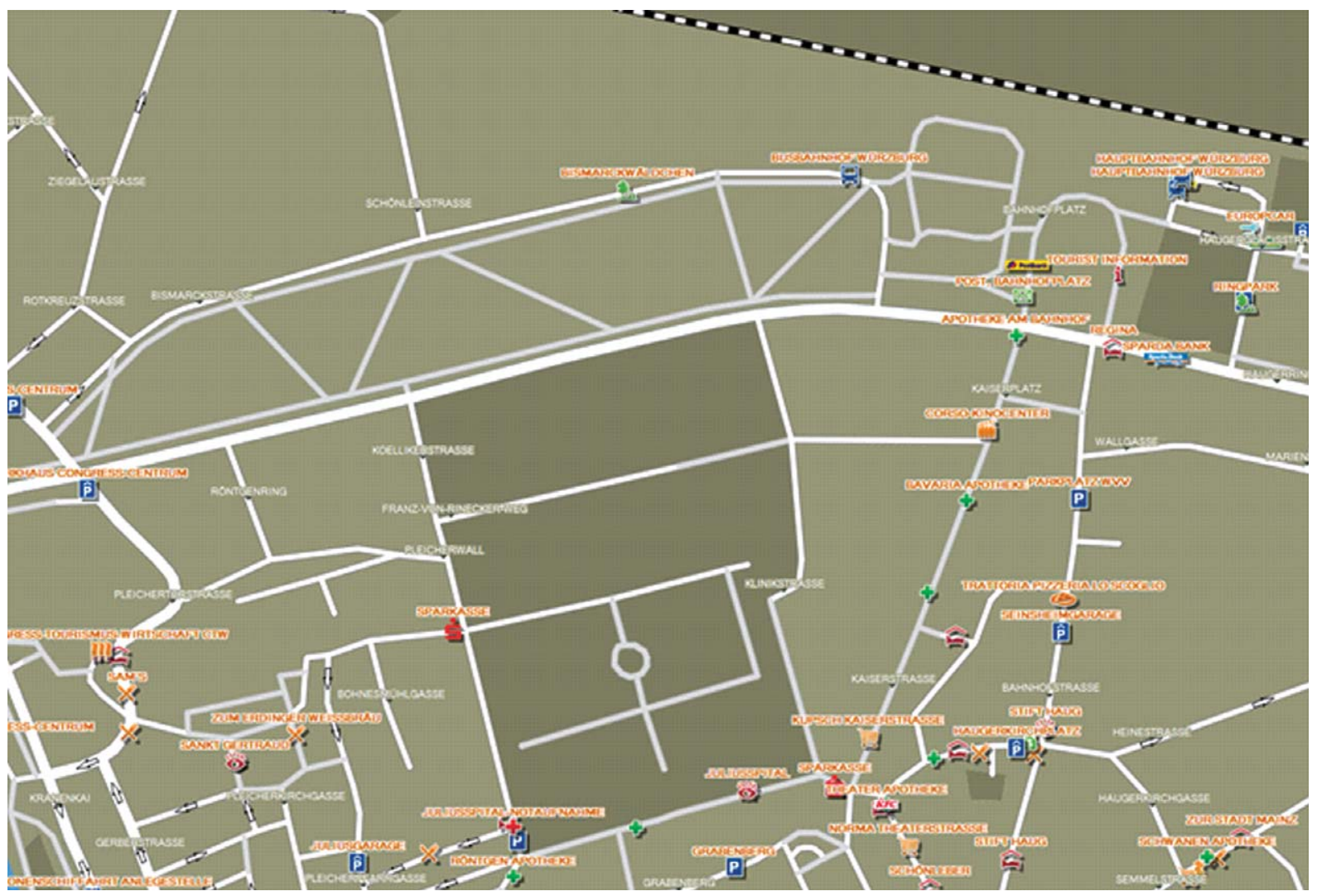

Fig. 9. Part of the test area Würzburg: map extended with pedetrian ways.

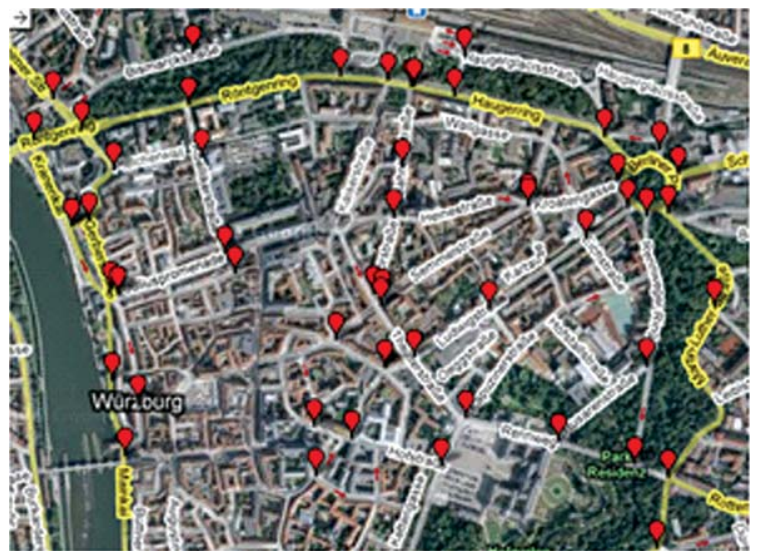

Fig. 10. Locations of digitized crosswalks.

availability of sidewalks, crosswalks, or stair ways, are not visualized in the digital map view.

Additional pedestrian attributes were provided as XML file including information on sidewalk locations, crosswalks, virtual connections, and stairways as

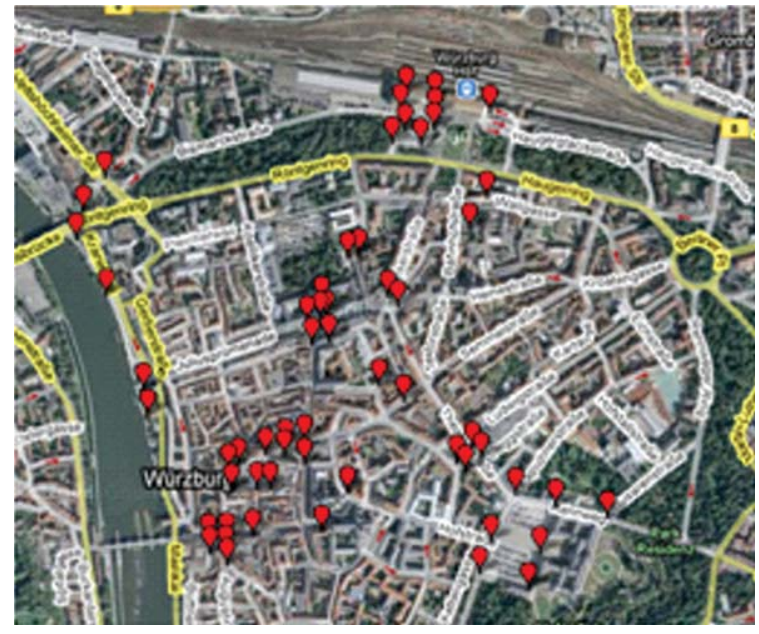

Fig. 11. Locations of virtual connections.

relational and geographical location information (see Figs. 10 and 11). Relational information is based on the internal street identifier and the object of interest. 
For instance, for each street section there is a unique identification number (BranchID) defined which is linked to the corresponding attributes. Usually a branch has many attributes. In the following example only two attributes are shown.

In the digital map's data model streets can have no (NONE), a left (LEFT), a right (RIGHT) or on both sides (LEFT_RIGHT) sidewalks (see Table 1). Similar to the sidewalk definition, stairs are represented by the attribute "StairLocation" given the value "MIDDLE". Crosswalks and pedestrian lights are defined as a relation between two linked street segments and a geo coordinate of the related junction.

An example representation of a pedestrian crossing with its relational description is illustrated in Fig. 12 and Table 2. Each corner of a junction is assigned with a BranchID of its adjacent street segment in clockwise direction. By this concept the location of a crosswalk or pedestrian light can be uniquely defined relative to the junction.

Although the additional attributes are originally intended to be used for pedestrian navigation, they also provide great benefit for the more complex task of scooter navigation. For instance, they allow the calculation of routes and navigation guidance for safety crossing of streets, avoiding of stairs, or dirty roads.

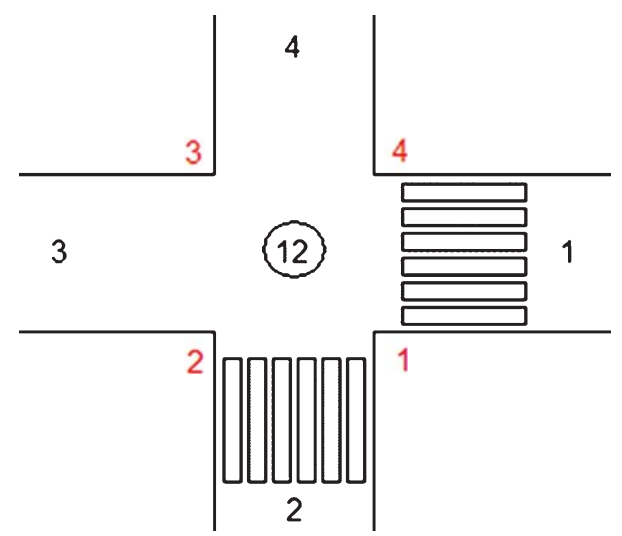

Fig. 12. Crosswalk representation.

Table 1

Attributes of a street segment

\begin{tabular}{lll}
\hline Branch id & Sidewalk location & Stair location \\
\hline 1 & LEFT_RIGHT & NONE \\
2 & LEFT & NONE \\
3 & NONE & MIDDLE \\
4 & RIGHT & NONE \\
\hline
\end{tabular}

Table 2

Relational representation of crosswalk

\begin{tabular}{lccc}
\hline Crossing ID & First branch ID & Second branch ID & Type \\
\hline 12 & 4 & 1 & ZEBRA \\
12 & 1 & 2 & ZEBRA \\
\hline
\end{tabular}

The basic idea of the developed routing algorithm is to use a weighted attribute based approach, such that stairs or other critical location are penalized (higher weights) to be excluded from the route. By using weights as penalizing concept the routing algorithm can also be fine tuned to calculate routes which might represent only suboptimal solutions. For instance, it is more reasonable to provide a route with just one stairway instead of forcing a pedestrian to walk a long detour. Within the Fit4Age project the existing navigation system was extended with an additional routing component [38] especially optimized for pedestrian route calculation based on the afore described pedestrian map attributes.

The following example illustrates the developed route calculation approach based on the pedestrian attributes and a penalty based routing algorithm. The task is to calculate a route for a Scooter from a selected start location to a target location without any barriers (like stairs). Start and end locations were chosen, such that the shortest route would contain a stairway (see Fig. 13, red circle).

For testing the stairs avoidance routing approach, a route was selected which includes at least one stair: Würzburg, Hofstallstraße 4 ->Würzburg, Neutorstraße 2 .

By weighting stairway attributes with the maximum penalty value, the routing algorithm calculated an alternative route (Fig. 14) which omitted the critical stairway route part.

As a result we can see that a safe route can be calculated by the use of the discussed attributes and routing extensions. As a consequence of obstacle avoidance the safe route is 118 meters longer than the shortest route. Depending on the user capabilities this can be an acceptable detour for avoiding critical situations.

Currently the routing algorithm is extended to take additional types of pedestrian attributes into consideration (e.g. crosswalks, sidewalks, and pedestrian lights) to increase safety. Possible use cases will comprise calculation of routes including safe crossing of streets and advise for choosing sidewalks on the appropriate street side. This demands ongoing research in finding 


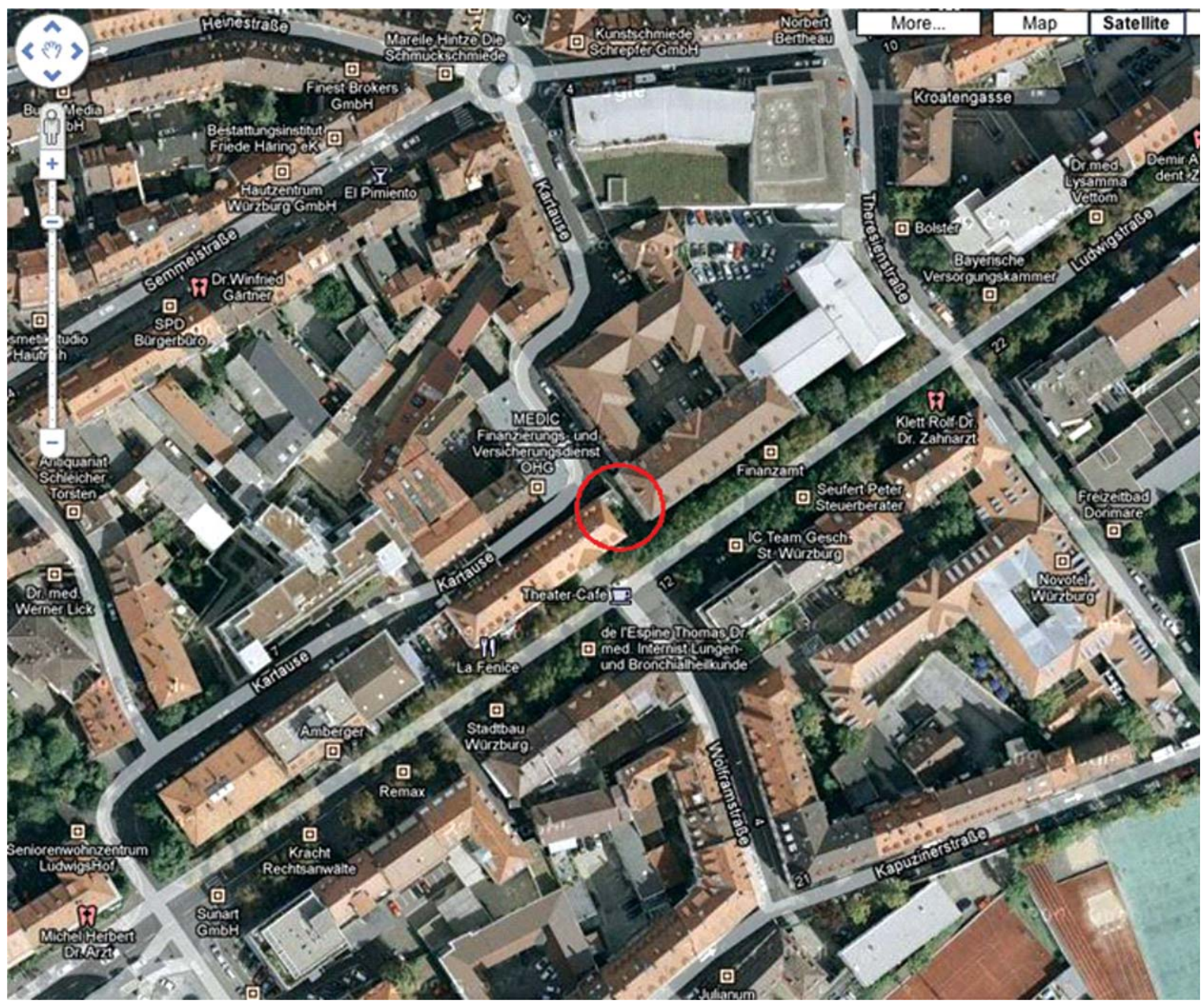

Fig. 13. Downtown of Würzburg, stairways in the red cycle [12].
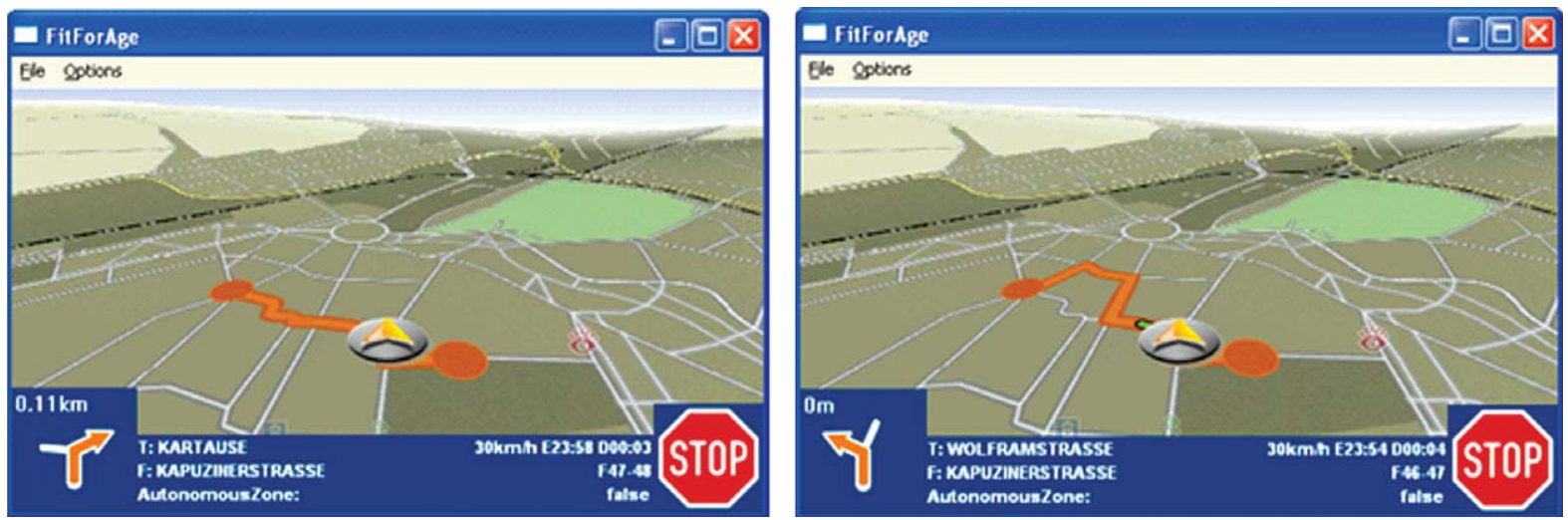

Fig. 14. Two example routes, left with no stairway penalty, right with stairway penalty. 
reasonable penalty coefficients with respect to trade off between optimal route detour length and safety. The integration of advanced positioning technologies will be a crucial factor for success.

\section{User studies: Evaluation and feedback loops with focus group methodology}

Following the participatory design approach, end users from a pre-to-target population were involved at various stages of the technical development process. One scope of this approach was to improve the quality and the functionality of the system continuously [16]. Considering that the single components of the mobility assistance system were in a rather early stage of product development and not fully implemented into the scooter model, focus group methodology was applied. The methodology is not meant to generate detailed feedback on the system quality or an in-depth evaluation of the system interface, but rather focuses on global user demands, preferences, and expectations towards the functionality and usability needs of such a mobility assistance system. In contrast to one-to-one interviews focus groups offer the possibility to get different opinions about particular system components discussed $[10,15]$. Following such considerations, the results reflect both multifaceted and varying user perspectives but also potential group consensus among users. Therefore, four groups with nine to ten participants each met in parallel generating more heterogeneous results.

\subsection{Method}

The 38 adults (13 female, mean age: $M=67.80$, $\mathrm{SD}=5.45$ ), who participated in the four focus groups were members of the Nuremberg-Erlangen Senior Counselling Board (SEN-PRO). In total, the SEN-PRO group includes 140 potential end users aged between 59 to 86 years that regularly participated in evaluation of prototypical products and services designed for an aging society [39]. Pilot studies as well as a literature review revealed that the involvement of health impaired users in early product development often produces misleading evaluation results $[26,35]$. Therefore, the sample was positively selected considering the participants' health and technical experience. About half of the members are highly educated and report expert knowledge in the use of modern information and communication technologies. Accordingly, none of the participants indicated to own or regularly use a scooter. However, one third of each group had experienced scooter usage in neighborhood or family before.

Each session of the four groups took at least three hours. The sessions started with an introduction to general conditions of scooter usage (e.g. target group, insurance issues, drivers' license). Afterwards, all participants were enabled to individually test the basic scooter model (see Fig. 2). We gave them a number of instructions that provided a broad insight into the system functionality. These included the initiation of the system, battery charging, a testing of the user-interface, the adjustment of the seat and the steering column, and a test on driving characteristics (brakes, acceleration, and navigation at bottlenecks). Two skilled student assistants recorded the feedback of participants during the entire testing session. After ending of this test phase, participants also reported their personal experiences in a group setting. In a next step of this procedure, participants worked with scenarios and possible functionalities of the single components of the mobility assistance system. Afterwards semistructured interviews were conducted to evaluate each system component with regard to potential target users, applications, strengths and weaknesses.

\subsection{Results}

The focus groups revealed insights regarding global user demands and expectations towards mobility assistance. In the following, the results of the four focus groups will be summarized with regard to single system components. Table 3 gives an overview of the respective feedback and the number of participants agreeing.

\subsubsection{Drive assistance system}

The participants discussed possible target groups and applications of the drive assistance system (as described in the section Hardware and software extensions of the scooter). For users with good physical abilities, the system was supposed to harbor the risk to take too much control of the driving situation. Nevertheless, single system components were considered assistive in certain use cases. During the test phase the participants identified a number of driving situations that are challenging especially for users with severe physical disabilities: reverse driving, bends, ascents, parking and bottlenecks. These situations require 
Table 3

Participants' feedback with regard to the single components of the mobility assistance

\begin{tabular}{lr}
\hline & $\begin{array}{c}\text { Number of } \\
\text { participants, } n(\%)\end{array}$ \\
\hline $\begin{array}{l}\text { Collision avoidance function } \\
\text { Decelerate rather than drive around } \\
\text { obstacles }\end{array}$ & $19(50.0)$ \\
Helpful for reverse driving & $9(23.7)$ \\
Deactivate with heavy traffic or crowded & $9(23.7)$ \\
$\quad$ places & \\
Assistance at bottlenecks & \\
Helpful for spatial confinements & \\
High additional costs & $19(50.0)$ \\
Parking assistance & $10(26.3)$ \\
High additional costs & \\
Acoustic warning system & $29(76.3)$ \\
Navigation system & $28(73.7)$ \\
Use on longer trips, vacations & \\
Use with orientation disorders & \\
Transportable & $28(73.7)$ \\
Compatible with scooter battery & $9(23.7)$ \\
\hline
\end{tabular}

sensitive control of speed and steering that are supposed to be controlled by the drive assistance system. Three drive assistance components (collision avoidance, navigation at bottlenecks, park assistance) were discussed in detail and shall therefore be explicitly referred to below.

The collision avoidance function was considered important for reverse driving as the visual field is limited during this driving situation and physically impaired users often have problems turning. As already referred to in section II, half of the users indicated that they generally prefer that the vehicle decreases in velocity instead of driving around obstacles. However, the system might be problematic to use in heavy or fast traffic situations as other vehicles might rear-end. Additionally, pedestrian zones with a huge number of potential obstacles to avoid, might lead to stop and go situations. Therefore, $23.7 \%$ of the participants argued to design the collision avoidance system as a function that can be easily deactivated.

During the user test, the participants reported difficulties in driving situations with spatial confinements like doors, elevators, bends, and corridors. Consequently, the idea of a system that assists the driver in navigating at narrows was positively evaluated by half of the participants. However, $23.7 \%$ of the senior adults feared extra costs that are disproportionate to the benefits of the system, as scooter usage still remains limited to barrier-free environments (e. g. operator control, access).

Parking was also seen as a challenging driving situation. Even so, $76.3 \%$ of the participants didn't speak in favor of an autonomous parking assistant as a respective system was supposed to cause high costs and to take too much control of the driving situation. Instead, the participants $(73.7 \%)$ proposed to implement an acoustic warning signal indicating the distance to surrounding obstacles.

\subsubsection{Scooter navigation system}

The scooter navigation system was discussed with respect to two different target groups. Most of the participants $(73.7 \%)$ highlighted challenges for average scooter users with physical disabilities. Handicapped users may know well where to find barrier-free routes, parking, and access to buildings in their immediate domestic environments. Nevertheless, such environments are often restricted to a limited radius. With the help of the navigation system, they might be more willing to extend their mobility radius and use the scooter also outside of well-known routes, for example, for a longer trip or a vacation. Besides route instructions, the system needs to provide information on barrier-free access to public buildings like restaurants, toilets, theaters, shops, and pharmacies and on walking tours that are suitable for scooter usage. A quarter of the focusgroup participants $(23.7 \%)$ also pointed to a possible other target group which may benefit from adjusting such a system to users with orientation disorders (e.g. MCI patients). The system might assist such target users to drive to predefined places and to find their way back home more easily. However, users with cognitive impairments might not be capable of learning how to use and finally accept the system, unless this has not been overlearned at an earlier, pre-symptomatic stage of the illness.

The focus groups also revealed insights into practical user demands. Such as that the navigation system needs to be transportable as one might wish to use it also as a pedestrian navigation system (26.3\% agreement). As separate battery recharging was seen as a chore, $23.7 \%$ of the participants suggested supplying the navigation system via the scooter battery.

In summary, the user studies helped identifying user demands and expectations with regard to the mobility assistance system. The results clearly show that a respective system is expected to assist the user in steering and speed control rather than to take complete 
control of the driving situation. Although the mobility assistance was in general positively evaluated, the participants expressed concerns about financial issues. Especially the drive assistance system was associated with high extra costs and might therefore not be affordable for some users. In addition, results show that barrier-free environment as identified by the scooter navigation system remain an important pre-condition for the use of scooters in various situations of daily living.

\section{Conclusion and future work}

This paper describes a scooter equipped with drive assistance function and a pedestrian navigation system extended with a seamless positioning by the means of GPS, WPS and INS to maintain the mobility of older people. Furthermore, the introduced system was evaluated in a user study with potential users. The feedback of the studies was already considered in the development phase of this project.

The basic mobility scooter and the hardware extension were introduced at the beginning. Several drive assistance functions, such as a velocity controller and a collision avoidance algorithm, were implemented on this vehicle. These assistance functions were always activated; hence no special human machine interface had to be integrated on the mobility scooter. The collision avoidance systems was developed according the requirements of the potential users in the user studies. However, the drive assistance functions support the operator and provide relief from the challenging control.

Due to the required high accuracy for the pedestrian navigation, the positioning system enhanced a GPS receiver with two further systems: WPS and INS. An interacting multiple model was implemented to achieve a seamless indoor and outdoor positioning. The results of the first implementation for the seamless localisation system were very satisfactory and enabled a pedestrian navigation on sidewalks and in shopping arcades.

Next to the mobility scooter, a navigation system was developed to support the mobility of the target group. For that purpose the digital map was extended with pedestrian specific attributes like sidewalks, stairs, crosswalks, pedestrian zones, and so on. The navigation system was further extended with an additional routing component, which determines routes with respect to the specific pedestrian attributes. The introduced positioning system was used to determine the position during the navigation. Finally, the navigation system was mounted on the mobility scooter. In this context, the GUI of the navigation system was adapted, according to the feedback of the user studies [37].

By means of the participatory design approach, in-depth utility and usability testing of the equipped scooter model is needed. Specific performance measure will clarify whether the presented systems will actually impact usage behaviour (e.g. in terms of fewer collisions, perceived safety). Finally, field studies will allow a better understanding of user satisfaction and acceptance.

In conclusion, the assistance systems support the operator of the mobility scooter and ease the handling of the vehicle. By the means of the navigation system, a safe route to the target can be determined, e.g. considering only safe street crossings. Altogether, such a vehicle maintains the mobility of older people and increases the safety on the way. Hence, the vehicle preserves a self-determined and independent life of the user.

In future, more drive assistance function will be integrated on the mobility scooter. Furthermore, some autonomous functions are planned for the future to further provide relief from the challenging control. The inertial sensor data of the positing system should be deeper incorporated to suppress position outliers from GPS or WPS in future. Even more promising is a tight coupling approach, combining the GPS pseudoranges and the RSSI-values from the WPS.

\section{Acknowledgments}

In the research association FitForAge (funded by the Bavarian Research Foundation) an interdisciplinary team of scientists and engineers from scientific and industrial institutions is cooperating. The aim of the research association is the development of technologybased solutions for older people assisting them in private as well as professional life.

\section{References}

[1] Awiloc: www.awiloc.com, 2012.

[2] Y. Bar-Shalom, X.-R. Li and T. Kirubarajan, Estimation with Applications to Tracking and Navigation, John Wiley and Sons Ltd., 2001. 
[3] L. Bergasa, R. Barea, E. Lopez, M. Escudero and J. Hernandez, Sira, a robotic system for elderly assistance, ISR 2004, (2004), 78-84

[4] H.A.P. Blom, An efficient filter for abruptly changing systems, Proceedings of the 23rd Conference on Decision and Control, Las Vegas, NV, December, 1984, 656-658.

[5] C. Chen, J. Ibanez-Guzman and O. Le-Marchand, Low-cost loosely-coupled GPS/odometer fusion: A pattern recognition aided approach, Information Fusion, 2008, 1-6.

[6] D. Ding and R.A. Cooper, Electric-Powered Wheelchairs, Control Systems Magazin, IEEE 25 (2005), 22-34.

[7] D. Eck, M. Stahl and K. Schilling, The Small Outdoor Rover MERLIN and its Assistance System for Tele-Operation, FSR, 2007.

[8] D. Eck, F. Leutert and K. Schilling, Development of a collision avoidance algorithm for a semi-autonomous scooter to support the mobility of elderly people, AAL-Congress, 2010.

[9] Federal Statistical Office, Germanys population until 2050 -11 th coordinated population projection, 2006, Wiesbaden, http://www.destatis.de/.

[10] A.D. Fisk, W.A. Rogers, N. Charness, S.J. Czaja and J. Sharit, Designing for older adults, 2nd edition, Boca Raton, Taylor and Francis FL, 2009.

[11] T. Gomi, A. Griffith, Developing intelligent wheelchairs for the handicapped, Assistive Technology and Artificial Intelligence, 1998, 150-178.

[12] Google: maps.google.de, 2012

[13] It2media, www.it2media.de à Products "MobileWalk", 2012.

[14] R.E. Kalman, A New Approach to Linear Filtering and Prediction Problems, Transaction of the ASME, Journal of Basic Engineering (1960), 35-45.

[15] R.A. Krueger and M.A. Casey, Focus groups: A practical guide for applied research, Thousand Oaks: Sage, 2008

[16] S. Kujala, User involvement: A review of the benefits and challenges, Behaviour and Information technology 22 (2003), $1-16$.

[17] V. Kulyukin, A. Kutiyanawala, E. LoPresti, J. Matthews and R. Simpson, Iwalker: Toward a rollator-mounted wayfinding system for the elderly, IEEE International Conference on RFID, 2008, 303-311.

[18] Layer.: www.layer.com, 2010.

[19] X.-R. Li and Y. Bar-Shalom, Performance prediction of the interacting multiple model algorithm, American Control Conference, 1992, 2109-2113.

[20] C. Martens, O. Ivlev, A. Gräser, Interactive controlled robotic system friend to assist disabled people, 7th International Conference On Rehabilitation Robotics (ICORR 2001), Evry, France, April 25-27, 2001, 148-154.

[21] S. Meyer, T. Vaupel and S. Haimerl, Wi-Fi coverage and propagation for localization purposes in permanently changing urban areas, IADIS International Conference Wireless Applications and Computing, 2008, 11-20.

[22] S. Meyer, Wo bin ich - Positionsbestimmung per WLAN, c'T Magazin, 2008, Heft 5, pp. 194-197.
[23] D. Miller and M. Slack, Design and testing of a low-cost robotic wheelchair prototype, Autonomous Robots 2 (1995), $77-88$.

[24] F. Müller, S. Meyer, S. Haimerl, T. Vaupel and K. Zahonyi, Ein skalierbares Umgebungsmodell für ortsabhängige Anwendungen, Sonderdruck Schriftenreihe der GeorgSimon-Ohm-Hochschule, Nürnberg, 2009.

[25] J.C. Nitz, Evidence from a cohort of able bodied adults to support the need for driver training for motorized scooters before community participation, Journal of Patient Education and Counseling 70 (2008), 276-280.

[26] R. Orpwood, S. Bjorneby, I. Hagen, O. Mäki, R. Faulkner and P. Topo, User involvement in dementia product development, Dementia 3 (2004), 263-279.

[27] M. Oussalah, Suboptimal multiple model filter for mobile robot localization, The International Journal of Robotics Research 20(12) (2001), 977-989.

[28] M. Pollack, L. Brown, D. Colbry, C. Orosz, B. Peintner, S Ramakrishnan, S. Engberg, J. Matthews, J. Dunbar-Jacob, C. McCarthy, S. Thrun, M. Montemerlo, J. Pineau and R. Roy, Pearl: A mobile robotic assistant for the elderly, AAAI, 2002.

[29] D. Rodriguez-Losada, F. Matia, A. Jimenez, R. Galan and G Lacey, Guido, the Robotic SmartWalker for the frail visually impaired, Proceedings of the first International Congress on Domestics, Robotics and Remote Assistance for All, 2005, 155-169.

[30] T. Röfer, T. Laue and B. Gersdorf, iWalker - An intelligent walker providing services for the elderly, Technically Assisted Rehabilitation, 2009.

[31] K. Schilling, H. Roth, R. Lieb and H. Stützle, Sensor supported driving aids for disabled wheelchair users, IFAC workshop on intelligent components for vehicles, Sevilla (1998), 267-270.

[32] A. Shumway-Cook and M.H. Woollacott, Motor control, Theory and Practical Applications, Philadelphia: Lippinkott Williams \& Wilkins, 2001.

[33] W.W. Spirduso, K.L. Francis and P.G. MacRae, Physical dimensions of aging, Campaign, IL Human Kinetics, 2005.

[34] Subhadra Evans, Claudius Neophytou, Lorraine de Souza, Andrew O. Frank, Young people's experiences using electric powered indoor - outdoor wheelchairs (EPIOCs): Potential for enhancing users' development? Disability and Rehabilitation Journal 26 (2007), 1281-1294 .

[35] S. Taylor, A new approach to empowering older people's forums: Identifying barriers to encourage participation, Practice 18 (2006), 117-128.

[36] U-blox.: www.u-blox.de, 2012.

[37] C. Uhlschmied, D. Eck, K. Schilling, I. Schens and B. Thomas, Safe navigation for people with degenerated physical and mental skills, AAL Congress Berlin, 2011.

[38] C. Uhlschmied, Scooter navigation und autonome steuerung auf gehwegen, Master Thesis, Universität Würzburg, Institut für Informatik, 2010.

[39] B. Williger and F.R. Lang, Technologie, Uni Kurier Magazin 35 (2009), 26. 

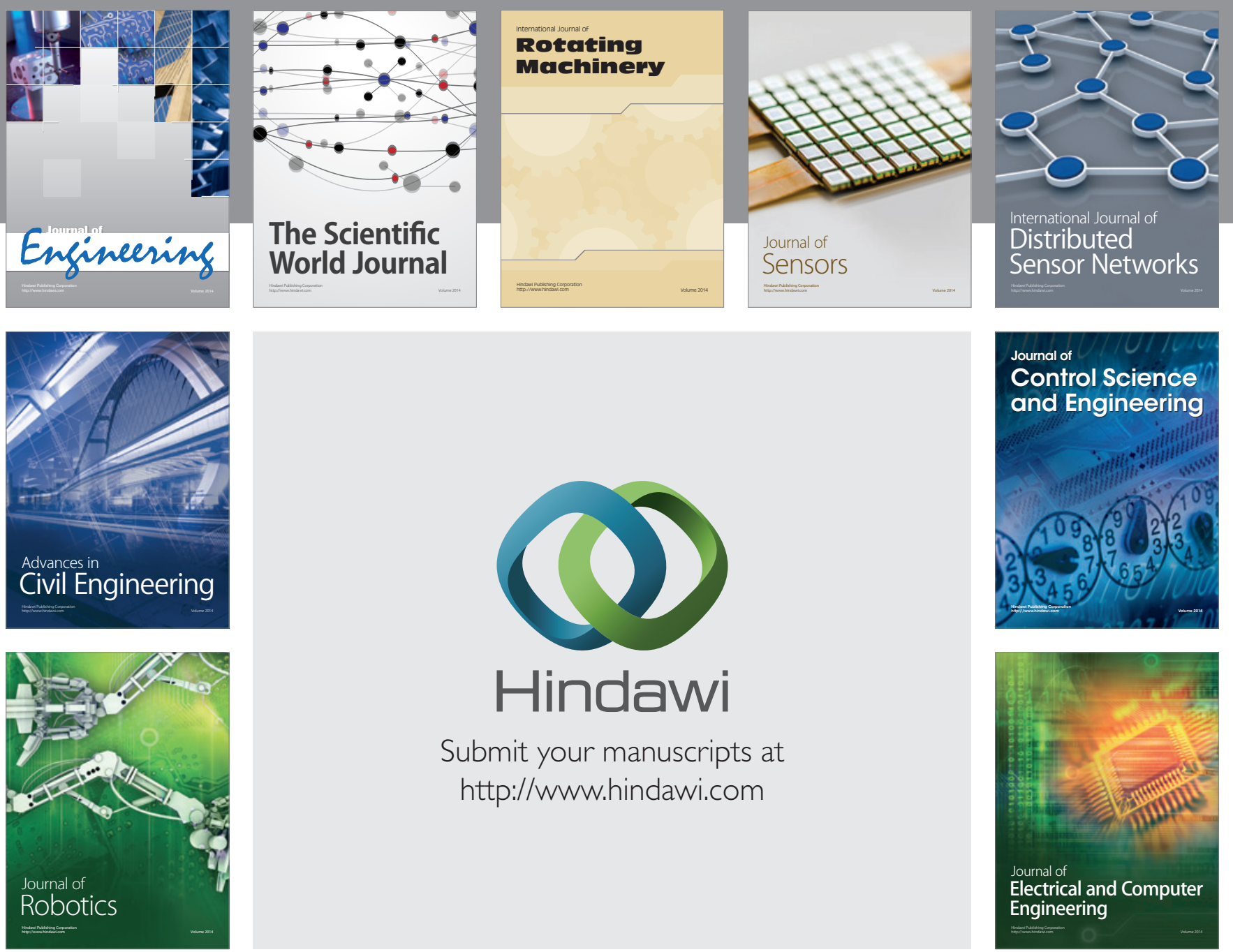

Submit your manuscripts at

http://www.hindawi.com
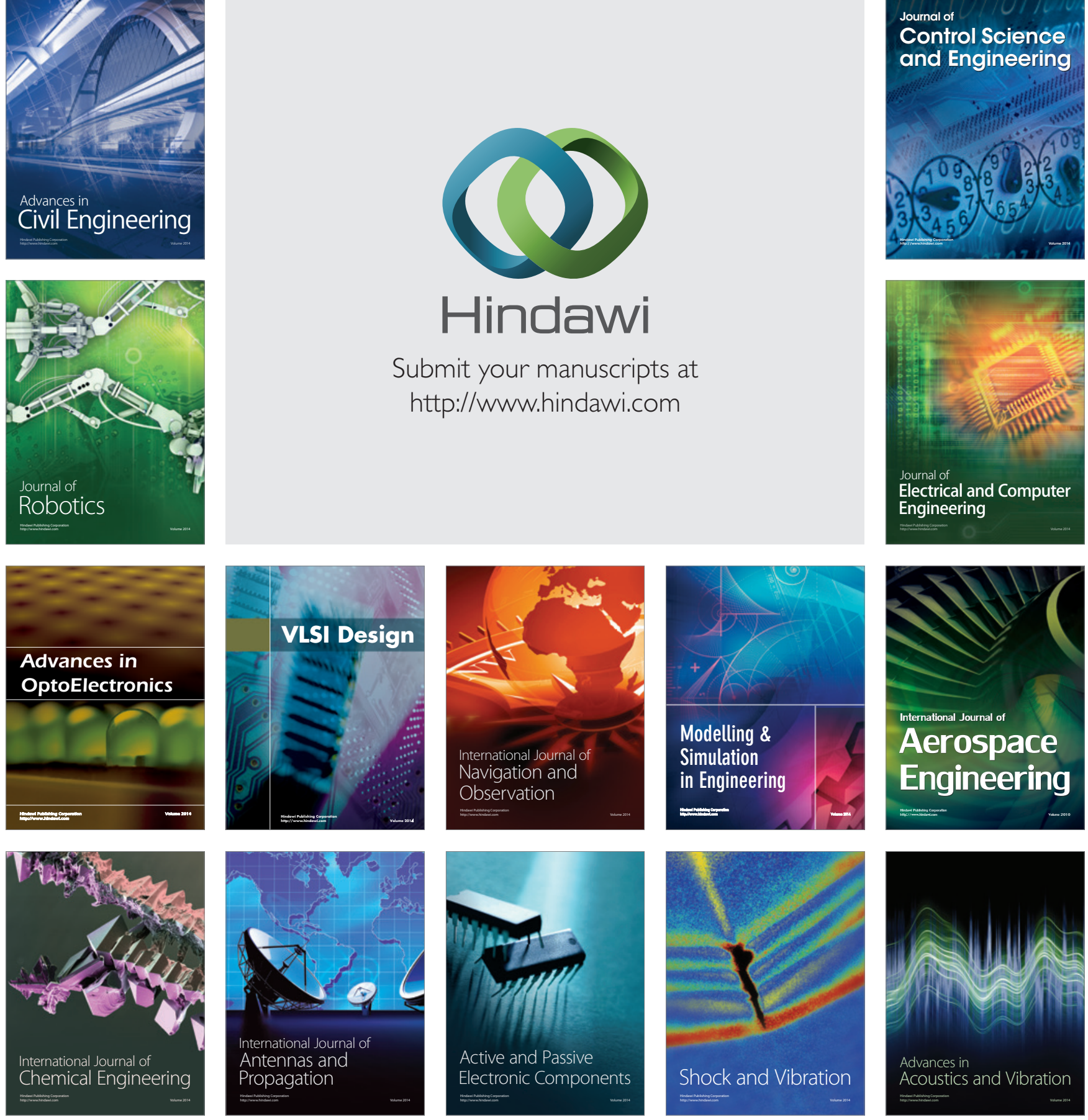